\title{
Barcelone (Espagne), 3-5 juin 2004 \\ Conférence internationale des présentateurs météo
}

0 rganisée par l'International Association of Broadcast Meteorology (IABM), la première conférence internationale des présentateurs météo s'est déroulée à Barcelone, du 3 au 5 juin 2004, dans le cadre du Forum universel des cultures (voir encadré). Plus de deux cents participants - météorologistes et présentateurs météo du monde entier - ont assisté à des exposés sur des thèmes tels que les modèles de prévision du temps et le rôle du prévisionniste, la gestion des catastrophes naturelles ou encore le changement climatique.

Sur ce dernier thème, les présentateurs météo ont souligné l'importance de leur profession et leur responsabilité vis-àvis du public qu'ils informent. Selon Gerald Fleming, ancien président de l'IABM et présentateur en Irlande, le changement climatique est préoccupant pour l'avenir de l'humanité. « Certaines dispositions sont difficiles et parfois contraignantes, mais elles sont nécessaires pour que nous réduisions les effets du changement climatique », a-t-il souligné. Pour Milagros Couchoud, directrice générale de l'Institut national météorologique (INM) espagnol, les météorologistes se doivent d'informer et d'éduquer le public de sorte qu'il comprenne la complexité des processus à

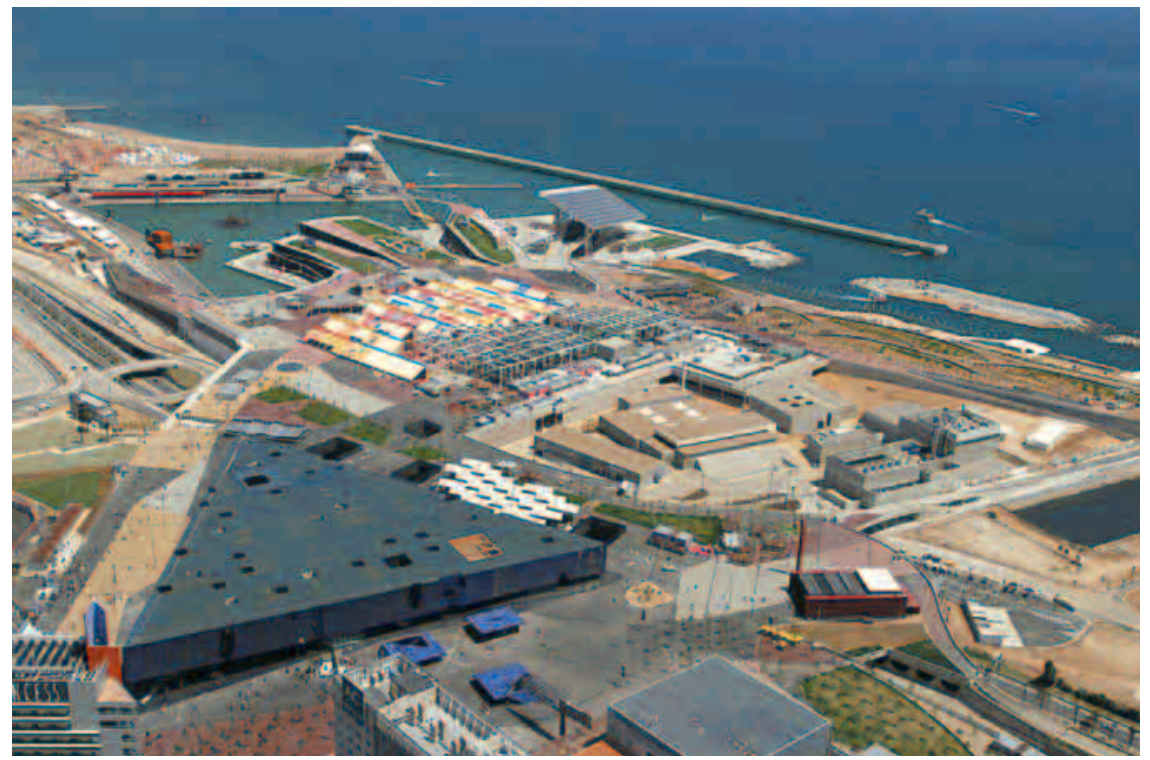

Le site central du Forum Barcelone 2004, vaste étendue de 30 hectares. (C Fòrum Barcelona 2004, Xavier M. Miró)

l'œuvre dans le changement climatique. Elle a ajouté qu'il était regrettable que, bien souvent, les gens ne s'intéressent aux prévisions météorologiques que lorsqu'elles sont en rapport avec leurs intérêts personnels. «Nous devons sensibiliser le public à l'importance des questions concernant l'atmosphère et le climat, car cet environnement a des répercussions majeures sur la société », a précisé la directrice de l'INM.
Lors de la réunion de presse d'ouverture de la conférence, Carine Van Maele, chef du Bureau de l'information de l'Organisation météorologique mondiale (OMM), a expliqué que l'un des principaux objectifs de l'OMM était d'améliorer la prévision des catastrophes d'origine météorologique et hydrologique : «En 2015, nous aurons réduit de manière significative le nombre de décès qui sont imputables aux

La présentation à la presse avant la conférence internationale des présentateurs météo. (C Fòrum Barcelona 2004, Xavier M. Miró)

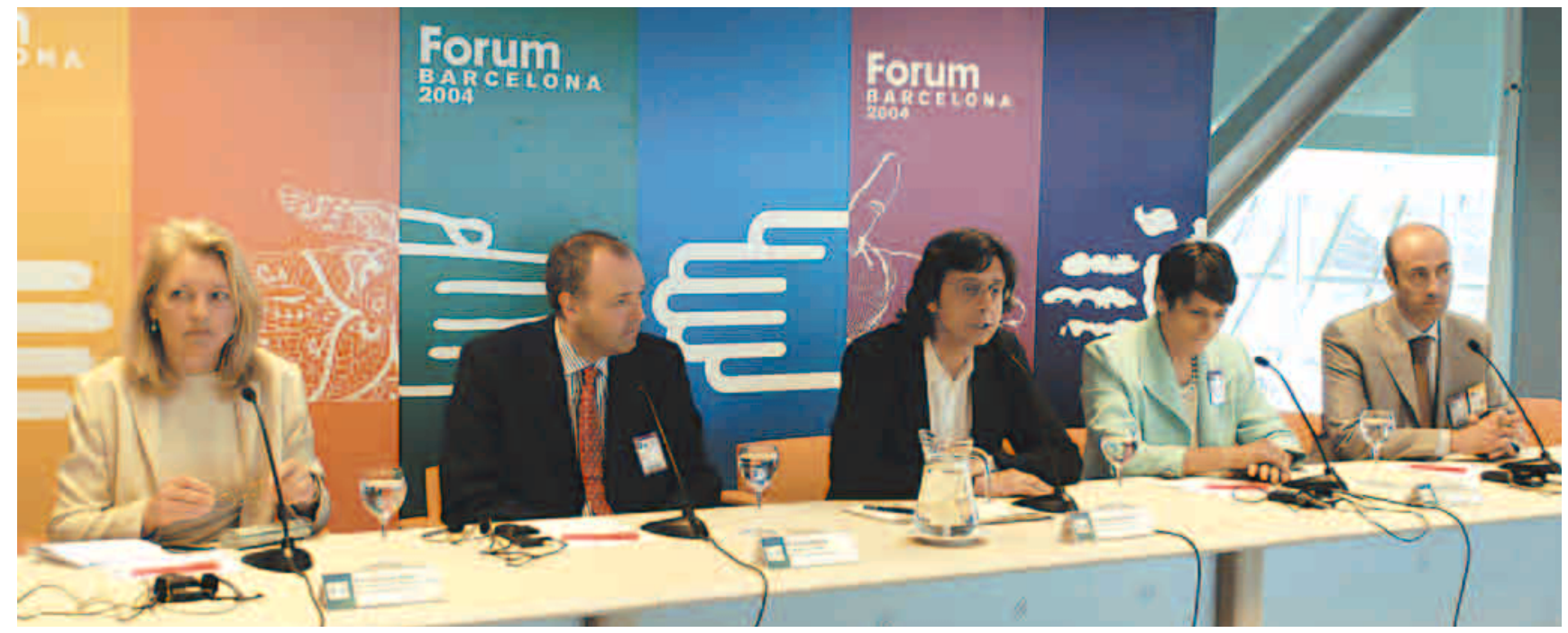


catastrophes naturelles ». L'information météorologique dans l'hémisphère sud est souvent inexistante, ce qui rend le travail des présentateurs météo plus difficile. Tomás Molina, météorologiste et présentateur météo en Catalogne, a indiqué que la conférence avait aussi pour but de favoriser le développement des services météorologiques dans les pays les plus exposés aux désastres naturels, où les moyens d'information sont souvent insuffisants. C'est dans cette optique que certains présentateurs météo en provenance de pays en voie de développement ont participé à un stage sur le thème de la communication météorologique au public.

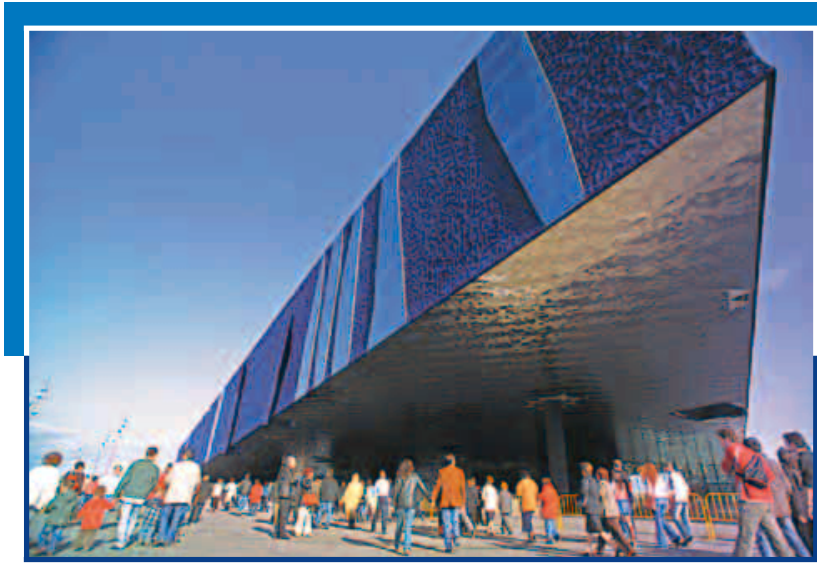

\section{Forum universel des cultures - Barcelone 2004}

Le Forum Barcelone 2004 (9 mai - 26 septembre 2004) est un événement international sous-tendu par trois grands axes thématiques : la diversité culturelle, le développement durable et les conditions de paix. Ces trois thèmes ont été couverts pendant toute la durée du forum sous la forme de spectacles, d'expositions, de conférences, de congrès et de manifestations invitant le public à la réflexion, mais aussi à la fête [www.barcelona2004.org].

L'une des réalisations architecturales du site central du Forum Barcelone 2004 (c) Fòrum Barcelona 2004, Agustí Argelich)
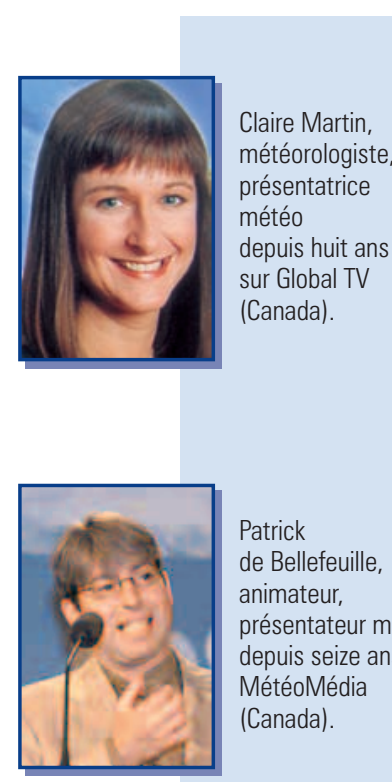

Patrick de Bellefeuille, animateur, présentateur météo Piernando Binaghi : Rencontrer des scientifiques, des chercheurs et, bien entendu, des collègues, pour

depuis seize ans sur échanger des expériences, prendre des informations et nouer des contacts. MétéoMédia (Canada).

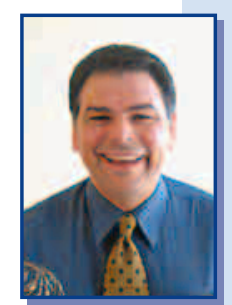

Franco Cavallaro, animateur, présentateur météo depuis seize ans sur CFCF-CTV (Canada).

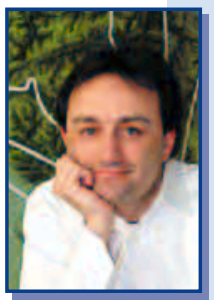

Piernando

Binaghi,

journaliste

scientifique,

présentateur météo depuis sept ans sur TSI (Suisse).

\section{Ouelques questions à...}

La Météorologie : Vous avez participé à la conférence internationale des présentateurs météo organisée par l'IABM à Barcelone du 3 au 5 juin derniers. Qu'en avez-vous pensé ?

Claire Martin : C'était une excellente conférence et une grande opportunité pour les météorologistes et les présentateurs météo du monde entier de se retrouver, de discuter de leur travail et des problèmes qu'ils ont en commun.

LM : Qu'attendez-vous de ce genre de colloque?

C. M. : Un point de vue global de la profession de présentateur météo. La météorologie n'a pas de frontières. Souvent, les participants des pays moins développés trouvent que ces colloques leur sont très utiles, ce qui, en soi, est plutôt gratifiant.

Patrick de Bellefeuille : La possibilité d'avoir accès à des données scientifiques pertinentes et de les utiliser ensuite dans mes bulletins afin que mon public soit informé au mieux sur les changements climatiques.

Franco Cavallaro : Apprendre davantage sur la météorologie, voir comment les autres pays présentent leur bulletin et échanger des idées avec mes collègues étrangers.

LM : Il a été dit que, ces dernières années, les catastrophes naturelles dues à des phénomènes météorologiques ont été multipliées par quatre. Que pensez-vous d'un tel constat?

P. d. B. : Je suis tout à fait d'accord. Si l'on ne fait pas quelque chose tout de suite, I'humanité risque d'être en péril. II est vraiment urgent d'agir.

P. B. : Je ne pense pas que cela soit vrai. Je suis très sceptique devant de tels propos. Nous n'avons pas assez d'informations et nous connaissons mal les liens directs entre catastrophes naturelles et changements climatiques. C'est souvent comme cela.

LM : Depuis que vous présentez le bulletin météo à la télévision, avez-vous constaté un changement dans la profession de présentateur météo?

C. M. : Le vrai changement dans la profession s'est produit au cours des cinq dernières années au Canada. De nos jours, il faut être plus qu'un présentateur, il faut aussi être un météorologiste. Ainsi, le vrai changement s'est fait à travers une approche plus scientifique des bulletins météo télévisés.

P. d. B. : Je trouve que le présentateur météo est davantage reconnu et mieux considéré qu'auparavant. Avec l'apparition du problème des changements climatiques, on le consulte plus fréquemment.

P. B. : Ce changement se traduit par une meilleure connaissance des risques possibles liés au climat. Les gens demandent davantage d'informations, mais le but n'est pas de leur dresser une vision catastrophique du futur. À mon avis, notre devoir est d'offrir une vision honnête et reposant sur les données scientifiques dont nous disposons, de mieux sensibiliser les gens à la maîtrise des énergies et aux gestes quotidiens qui permettent de réduire les émissions de gaz à effet de serre.

F. C. : Le travail du présentateur météo a changé, grâce aux nouvelles technologies d'information et de communication, par exemple Internet. Dans mon bulletin, je privilégie le professionnalisme tout en délivrant un message compréhensible par tous, auquel il m'arrive d'ajouter une pointe d'humour quand c'est opportun. 


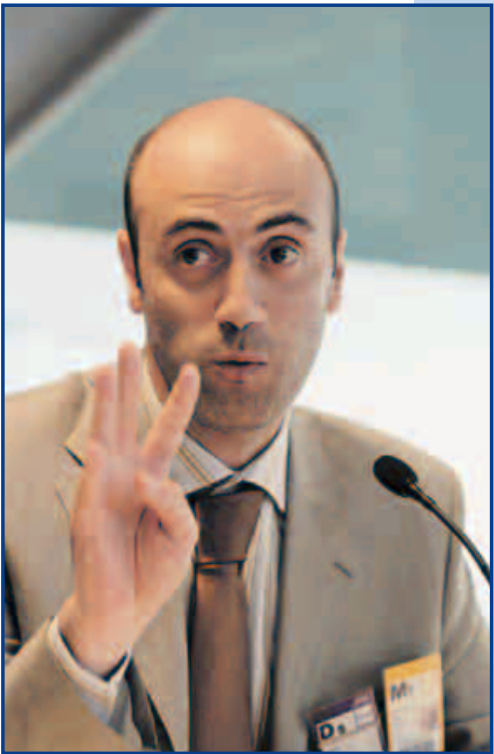

Tomás Molina

(c) Fòrum Barcelona 2004.

Xavier M. Miró)

\section{Tomás Molina,}

nouveau président de I'IABM

L'IABM a été créée en 1994 avec pour objectif de rassembler les présentateurs météo et de leur permettre de s'exprimer collectivement. C'est à Barcelone, en parallèle de la conférence, que l'IABM a tenu son assemblée générale 2004. Tomás Molina a succédé à Gerald Fleming à la présidence de l'association.

La Météorologie : L'IABM regroupe les présentateurs météo d'une trentaine de pays. Faut-il avoir nécessairement une formation de météorologiste pour adhérer à votre association?

Tomás Molina : L'IABM est une association de présentateurs météo, c'est-à-dire une association professionnelle pour ceux qui travaillent dans les domaines de la météorologie et des médias. Parmi nos adhérents, il y a beaucoup de présentateurs météo, mais également des producteurs météo et même des sociétés météorologiques privées. Nous avons également comme membres associés des services météorologiques et des chaînes de télévision.

LM : Lors de la conférence de Barcelone, certaines des interventions étaient d'un niveau scientifique très élevé. Pensez-vous que ce genre d'interventions puisse intéresser les présentateurs météo de formation non scientifique ou bien sont-elles destinées exclusivement aux météorologistes?

T. M. : Notre travail, que ce soit dans les médias ou dans les sociétés qui fournissent des informations météorologiques, est d'aller vers le public avec les meilleures informations et prévisions disponibles. Cela signifie que nous devons nous tenir informés des dernières évolutions en météorologie. Nous n'avons pas besoin d'être les meilleurs scientifiques, mais nous devons connaître les techniques de prévision, la variabilité de notre climat et la vulnérabilité du public. Nous jouons un rôle important dans la sensibilisation du public en matière de climat et nous avons un rôle clé dans la sécurité des personnes et des biens en cas de mauvaises conditions météorologiques. Je suis convaincu, et nous avons été très bien accueillis sur ce point par la plupart des participants de la conférence de Barcelone, qu'un certain niveau scientifique est nécessaire dans n'importe quelle conférence de météorologie journalistique.

LM : Avez-vous l'intention d'organiser d'autres conférences comme celle de Barcelone ?

T. M. : Oui. L'organisation du Forum universel des cultures de Barcelone a valorisé notre congrès et en a fait l'un des objectifs principaux de l'événement général. II sera d'ailleurs à l'ordre du jour qui sera proposé pour le prochain forum universel qui doit se dérouler à Monterrey, au Mexique. En attendant, nous participerons, en tant que partenaire ou sponsor, à d'autres conférences telles que celles de l'EMS européenne, de l'AMS américaine ou de la RMS britannique. Nous serons heureux de participer aussi au Forum international de la météo à Paris. Le Festival de météo a été un grand succès et notre association est née au cours de l'une des éditions du festival à Issy-les-Moulineaux. Nous sommes très reconnaissants au Festival, à son fondateur François Fandeux et à toute son équipe.

LM : Envisagez-vous des collaborations avec d'autres associations ou organismes pour des conférences ou des formations à destination des pays en voie de développement ? T. M. : Nous sommes très actifs en ce qui concerne l'aide aux pays en voie de développement. Nous proposons une adhésion gratuite aux membres de ces pays. Nous avons participé et organisé de nombreuses fois des ateliers de formation un peu partout dans le monde. Nous avons reçu le statut d'observateur auprès de l'OMM et nous travaillons très étroitement avec elle et les services météorologiques nationaux pour adapter les ateliers aux besoins des présentateurs, des populations et des médias.

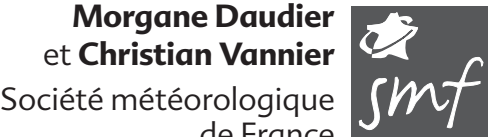

de France 